\title{
Tensely strained silicon on SiGe produced by strain transfer
}

\author{
D. Buca, ${ }^{a}$ B. Holländer, H. Trinkaus, and S. Mantl \\ Institut für Schichten und Grenzflächen, and cni-Center of Nanoelectronic Systems for Information \\ Technology, Forschungszentrum Jülich, D-52425 Jülich, Germany \\ R. Carius \\ Institut für Photovoltaik, Forschungszentrum Jülich, D-52425 Jülich, Germany \\ R. Loo and M. Caymax \\ IMEC, Kapeldreef 75, B-3001, Leuven, Belgium \\ H. Schaefer \\ Infineon Technologies AG, Otto-Hahn-Ring 6, 81739, München, Germany
}

(Received 18 June 2004; accepted 19 July 2004)

\begin{abstract}
An approach for the controlled formation of thin strained silicon layers based on strain transfer in an epitaxial $\mathrm{Si} / \mathrm{SiGe} / \mathrm{Si}(100)$ heterostructure during the relaxation of the $\mathrm{SiGe}$ layer is established. $\mathrm{He}^{+}$ion implantation and annealing is employed to initiate the relaxation process. The strain transfer between the two epilayers is explained as an inverse strain relaxation which we modeled in terms of the propagation of the dislocations through the layers. Effcient strain buildup in the Si top layer strongly depends on the Si top layer thickness and on the relaxation degree of the SiGe buffer. $100 \%$ strain transfer was observed up to a critical thickness of the strained silicon layer of $8 \mathrm{~nm}$ for a $150 \mathrm{~nm}$ relaxed $\mathrm{Si}_{0.74} \mathrm{Ge}_{0.26}$ buffer. () 2004 American Institute of Physics.
\end{abstract}

[DOI: $10.1063 / 1.1790593$ ]

This letter presents a process for strain transfer within an epitaxial $\mathrm{Si} / \mathrm{SiGe} / \mathrm{Si}(100)$ heterosystem and the involved theoretical rationale. As strained silicon $(\mathrm{sSi})$ permits a significant improvement of nanoelectronic devices, due to a large enhancement of the mobility of the electrons and holes, this material presently receives enormous interest. ${ }^{1}$ Our approach permits the formation of a sSi layer by epitaxial growth of a thin $\sim 200 \mathrm{~nm}$ heterosystem, unlike the most common approach, which involves the growth of a thick (several $\mu \mathrm{m}$ ) relaxed $\mathrm{SiGe}$ buffer layer, and the polishing and overgrowth of the strained layer. ${ }^{2}$ Here, a cubic $\mathrm{Si} /$ strained $\mathrm{SiGe} / \mathrm{Si}(100)$ heterostructure is formed first. Then the elastic energy stored in the pseudomorphic $\mathrm{Si}_{1-x} \mathrm{Ge}_{x}$ layer is relaxed in a controlled way to transform the cubic top layer into $\mathrm{sSi}$. In order to initiate this inverse strain relaxation process a $\mathrm{He}^{+}$implantation into the $\mathrm{Si}(100)$ substrate and an annealing step are conducted. During this process a narrow defect band underneath the $\mathrm{SiGe} / \mathrm{Si}$ substrate interface is generated. It provides a high density of dislocation loops as sources for misfit dislocations (MDs) yielding efficient strain relaxation during annealing with low densities of threading dislocations (TDs). ${ }^{3,4}$ For the strain transfer we make use of the propagation of the dislocations from the bottom of the relaxing SiGe layer towards the surface into the Si cap. This process differs from purely thermally induced strain relaxation where low densities of dislocations are nucleated randomly at the surface. We model the strain buildup in the Si layer by considering the forces acting on the dislocations and their resulting motion through the layers in detail. Our approach also differs from strain transfer between a film and a compliant substrate where only limited areas can be transformed and the misfit strain is diluted due to the elastic energy balance the two layers. ${ }^{5}$

\footnotetext{
a) Author to whom correspondence should be addressed; electronic mail:
} d.m.buca@fz-juelich.de
We have studied the strain transfer between $\mathrm{Si}_{1-x} \mathrm{Ge}_{x}$ buffers and $\mathrm{Si}$ top layers as a function of $\mathrm{Si}$ film thickness. The $\mathrm{Si} / \mathrm{Si}_{1-x} \mathrm{Ge}_{x}$ heterostructures were grown by chemical vapor deposition (CVD) on 150 and $200 \mathrm{~mm} n$ - and $p$-type $\mathrm{Si}(100)$ wafers in different CVD reactors. All wafers were implanted with $7 \times 10^{15} \mathrm{He}^{+} / \mathrm{cm}^{2}$ with an energy of $30-50 \mathrm{keV}$, such that ions stopped about $150 \mathrm{~nm}$ below the $\mathrm{SiGe} / \mathrm{Si}$ substrate interface. Then the samples were annealed at $850^{\circ} \mathrm{C}$ for $10 \mathrm{~min}$ in $\mathrm{Ar}$ flow. It is important to note that the annealing produces interdiffusion of only $1 \mathrm{~nm} .{ }^{6}$

The strain in the $\mathrm{Si}$ and $\mathrm{SiGe}$ epilayers was analyzed by Raman spectroscopy. ${ }^{1,7,8}$ We use a wavelength of $415 \mathrm{~nm}$ for excitation at low laser-power density. No interference effects from the underlying silicon substrates were observed. We used the expressions given in Ref. 8 to calculate the $\mathrm{Si}-\mathrm{Si}$ optical phonon frequency, $\omega_{1}$, in relaxed $\mathrm{Si}_{1-x} \mathrm{Ge}_{x}$. The degree of relaxation of the $\mathrm{Si}_{1-x} \mathrm{Ge}_{x}$ layer, $R$, is calculated as the ratio of the frequency change due to the actual relaxation, $\omega_{r}-\omega_{0}$, to the corresponding difference for full relaxation, $\omega_{1}-\omega_{0}$, i.e., $R=\omega_{r}-\omega_{0} / \omega_{1}-\omega_{0}$, where $\omega_{0}$ is the $\mathrm{Si}-\mathrm{Si}$ optical phonon frequency for pseudomorphic $\mathrm{Si}_{1-x} \mathrm{Ge}_{x}$ layers.

Elastic strain buildup in the $\mathrm{Si}$ layers and simultaneous strain relaxation of the SiGe layers are shown in Fig. 1 by the

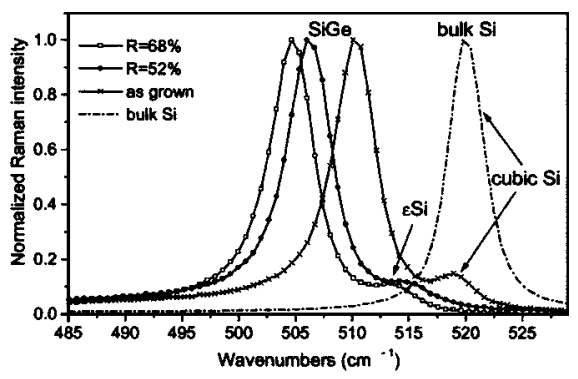

FIG. 1. Raman spectra of $\mathrm{Si}-\mathrm{Si}$ modes from $\mathrm{SiGe}$ and $\mathrm{sSi}$ epitaxial films of a $\mathrm{Si} / \mathrm{Si}_{0.71} \mathrm{Ge}_{0.29} / \mathrm{Si}$ heterostructure after growth and different strain relaxations. The bulk Si spectrum is shown for reference. 
Raman spectra of an as-grown and two differently relaxed SiGe layers $(R=52 \%$ and $68 \%)$. The heterostructure consists of $145 \mathrm{~nm} \mathrm{Si}{ }_{071} \mathrm{Ge}_{0.29}$ and a $5 \mathrm{~nm} \mathrm{Si}$ top layer. The presence of the deconvoluted Si peak at $520 \mathrm{~cm}^{-1}$ confirms the cubic structure of the Si film in the as-grown state. The peak shift of the $\mathrm{Si}$ modes in the $\mathrm{Si}$ layers, resulting from the $\mathrm{Si}_{0.71} \mathrm{Ge}_{0.29}$ layer relaxation, reveals the tensile strain in the film. The wave-number shift was converted to elastic strain $\varepsilon_{\mathrm{Si}}$ using an expression given in Ref. 7

For further discussion, we introduce parameters characterizing strain relaxation and generation in the $\mathrm{SiGe}$ and $\mathrm{Si}$ top layer, respectively. The change in the strain of the SiGe layer upon relaxation may be expressed by the relaxation degree, $R$, as $\Delta \varepsilon_{\mathrm{SiGe}}=R x f_{\mathrm{Ge}, \mathrm{Si}}$, where $f_{\mathrm{Ge}, \mathrm{Si}}=4.2 \%$ is the mismatch between the Ge and the Si lattice and $x$ is the Ge content in at. \%. To quantify the efficiency of the strain transfer we define the ratio of the strain induced in the Si top layer, $\varepsilon_{\mathrm{Si}}$, to the strain change in the SiGe layer, $\Delta \varepsilon_{\mathrm{SiGe}}$, as the strain transfer efficiency $\eta$, such that $\varepsilon_{\mathrm{Si}}$ may be written as $\varepsilon_{\mathrm{Si}}=\eta \Delta \varepsilon_{\mathrm{SiGe}}=\eta R x f_{\mathrm{Ge}, \mathrm{Si}}$. Full strain relaxation $(R$ $=100 \%$ ) and ideal strain transfer of $\eta=100 \%$ would result in a maximum in-plane strain in the silicon cap layer, i.e., $\varepsilon_{\mathrm{Si}}$ $=1.21 \%$ for a $\mathrm{Si}_{0.71} \mathrm{Ge}_{0.29}$ layer. For $R=68 \%$ of the $\mathrm{SiGe}$ layer we measured $\varepsilon_{\mathrm{Si}}=0.85 \%$ in the $5 \mathrm{~nm} \mathrm{Si}$ layer which corresponds to strain transfer efficiency of $100 \%$. Obviously, the Si film follows perfectly the change in the in-plane lattice constant of the $\mathrm{Si}_{0.71} \mathrm{Ge}_{0.29}$ layer. Deconvolution of the $\mathrm{sSi}$ and $\mathrm{SiGe}$ signals results in a negligible width change of the sSi peak indicating a uniform strain distribution in the $\mathrm{sSi}$ layer.

In order to investigate the strain transfer systematically we also studied $\mathrm{Si} / \mathrm{Si}_{0.74} \mathrm{Ge}_{0.26}$ heterostructures with a buffer thicknesses of $150 \mathrm{~nm}$ and $\mathrm{Si}$ cap thicknesses $d_{S i}$ up to $30 \mathrm{~nm}$. For comparison of the strain accumulated in the $\mathrm{Si}$ layer versus its thickness we first analyzed the relaxation of the $\mathrm{Si}_{1-x} \mathrm{Ge}_{x}$ layers as a function of $\mathrm{Si}$ layer thickness. We found that $\mathrm{Si}_{0.74} \mathrm{Ge}_{0.26}$ layers relaxed independently of the top Si layer thickness.

Our data indicate that the main parameters controlling strain generation in the Si top layer are its thickness, $d_{\mathrm{Si}}$, and the degree of relaxation of the SiGe layer, $R$ (more precisely the change in the strain upon relaxation in the SiGe layer, $\left.\Delta \varepsilon_{\mathrm{SiGe}}\right)$. On the basis of the reciprocal relationship between the critical thickness and the strain in all existing strain relaxation models we plot the data of $\mathrm{Si}$ strain, $\varepsilon_{\mathrm{Si}}$, versus reciprocal Si thickness $\left(1 / d_{\mathrm{Si}}\right)$ as shown in Fig. 2. At small $1 / d_{\mathrm{Si}}\left(d_{\mathrm{Si}}>8 \mathrm{~nm}\right)$, the data for all degrees of SiGe relaxation lie close to one single straight line intersecting the $1 / d_{\mathrm{Si}}$ axis. This observation indicates a relationship of the form

$$
\varepsilon_{\mathrm{Si}}=A\left(\frac{1}{d_{\mathrm{Si}}}-\frac{1}{d^{*}}\right), \quad d_{\mathrm{Si}}<d^{*}
$$

where $A$ and $d^{*}$ are parameters for which linear regression yielded $8.16 \times 10^{-2}$ and $59 \mathrm{~nm}$, respectively, apparently independent of the degree of relaxation of the SiGe layer (inclined line in Fig. 2). At large $1 / d_{\mathrm{Si}}\left(d_{\mathrm{Si}}<8 \mathrm{~nm}\right)$, on the other hand, the strain $\varepsilon_{\mathrm{Si}}$ seems to saturate at a level determined by the relaxation degree of the SiGe layer $(\eta=100 \%$, i.e., $\varepsilon_{\mathrm{Si}}=\Delta \varepsilon_{\mathrm{SiGe}}$ is used for the horizontal lines in Fig. 2).

In the following, we make an attempt to rationalize these results in terms of the formation of the dislocation loops in

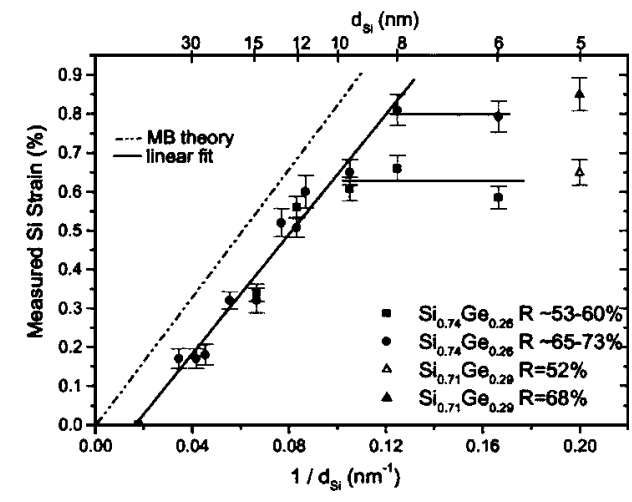

FIG. 2. Measured strain in the top Si layer vs reciprocal Si layer thickness for a series of heterostructures with different $R$. The inclined and horizontal solid lines represent fits to experimental data corresponding to $\eta<0.95$ and $\eta>0.95$, respectively. The dashed line corresponds to the critical thickness relation according to $\mathrm{MB}$ (Ref. 9).

the Si-substrate/SiGe interface, the extension of the segments of these loops towards the SiGe/top Si interface ${ }^{10}$ and the $\mathrm{Si}$ surface and the spreading of the TDs through both layers. As shown previously, He-filled cavities, underneath the SiGe layer are responsible for the formation of dislocation loops which glide towards the interface. ${ }^{4}$ Under compressive plane stress in SiGe, one segment of such a loop is held at the interface where it forms a MD segment while the other one is driven through the $\mathrm{SiGe}$ layer towards the $\mathrm{SiGe} / \mathrm{Si}$ top interface.

When a dislocation segment has passed the interface, the surface image force pulling it towards the surface must be sufficiently strong to overcome the counter force resulting from the fact that $\mathrm{Si}$ is elastically stronger than $\mathrm{SiGe}$ and from the tensile plane stress already built up in the top $\mathrm{Si}$ layer. Equilibrium between these three forces suggests a condition of a form equivalent to that defined by Eq. (1). For this intermediate stage of the deformation process, the term corresponding to $A / d_{\mathrm{Si}}$ in Eq. (1) represents the strength of the surface image force and $d^{*}$ the critical thickness $(59 \mathrm{~nm}$ according to our data fit) above which this force is no longer able to pull the dislocation away from the interface.

Once a dislocation loop has reached the surface of the $\mathrm{Si}$ top layer, the two TD arms connecting the MD segments in the $\mathrm{SiGe} / \mathrm{Si}$-substrate interface with the surface will spread to both sides, depending on the specific conditions as will be discussed in detail in the following (see Fig. 3). During the motion of a TD three types of forces are in stationary equi-
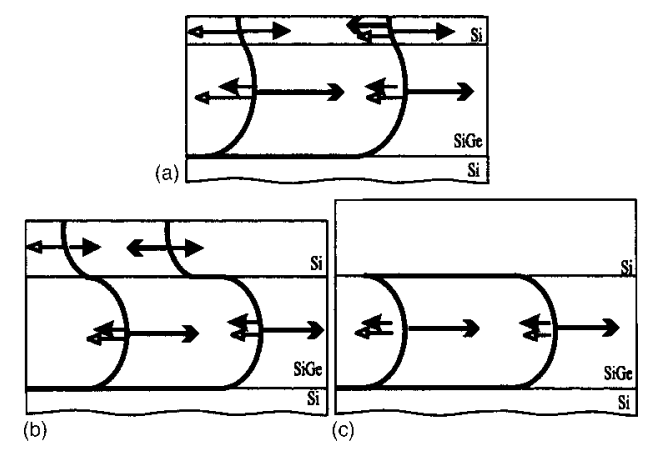

FIG. 3. Sketch of different situations in the motion of TDs through a SiGe buffer and a Si top layer. (a), (b), and (c) illustrate complete $\left(\varepsilon_{\mathrm{Si}}=\Delta \varepsilon_{\mathrm{SiGe}}\right)$, partial $\left(0<\varepsilon_{\mathrm{Si}}<\Delta \varepsilon_{\mathrm{SiGe}}\right)$, and vanishing $\left(\varepsilon_{\mathrm{Si}}=0\right)$ plastic strain transfer, respectively. Full thick, full thin, and open arrows indicate forces due to stress,

line tension, and friction, respectively.

to AIP license or copyright, see http://apl.aip.org/apl/copyright.jsp 
librium with each other: forces due to stress, line tension, and friction. In the SiGe layer, the TD motion is driven by the compressive stress counteracted by line tension and frictional forces. In the $\mathrm{Si}$ top layer, the curvature of the TD, being tracked by its segment in SiGe layer, changes its sign such that the driving force for its motion becomes the line tension force, counteracted by forces due to friction and the tensile stress being built up.

For small $d_{\mathrm{Si}}$ [Fig. 3(a)], the segment of the TD within the top $\mathrm{Si}$ is able to follow that in the SiGe layer during the whole deformation process because of the large line tension force associated with its large curvature, allowing complete plastic strain transfer or $100 \%$ strain-transfer efficiency $(\eta$ $=100 \%, \varepsilon \mathrm{Si}=\Delta \varepsilon_{\mathrm{SiGe}}$ ). As shown in Fig. 2, this ideal regime extends up to a top Si layer thicknesses of about $8 \mathrm{~nm}$. For nanoelectronic applications such a sSi layer bonded on a second oxidized wafer is thick enough for the realization of ultrathin silicon-on-insulator metal-oxide-semiconductor field-effect transistors. ${ }^{11}$ It can be used also as a Si seed layer for optional epitaxial overgrowth.

In an intermediate range of $d_{\mathrm{Si}}$ values [Fig. 3(b)], the segment of a TD within the Si top layer follows the SiGe TD segment only at the beginning of the deformation process, but falls back when the line tension force (then maximum) is compensated by the force due to the tensile stress built up. This transition occurs at a critical strain $\varepsilon_{\mathrm{Si}}$, independent of the further relaxation of the SiGe layer, determined by the critical thickness relation according to Matthews and Blakeslee (MB). ${ }^{9}$

$$
\varepsilon_{\mathrm{Si}} d_{\mathrm{Si}}=\frac{\left(1-v\left\langle\cos ^{2} \Theta\right\rangle\right)}{8 \pi(1+v) \cos \lambda} b \ln \left(\frac{\alpha d_{\mathrm{Si}}}{b}\right),
$$

where $v$ is Poisson's ratio, $\Theta$ is the angle between the Burgers vector and the dislocation line, $\lambda$ is the angle between the Burgers vector and the normal to the dislocation line in the interface, $b$ is the magnitude of the Burgers vector, and $\alpha$ is the dislocation core parameter. Using $v=0.23, \quad b$ $=0.38 \mathrm{~nm},\left\langle\cos ^{2} \Theta\right\rangle=1 / 2, \lambda=60^{\circ}$, and $\alpha=2(1<\alpha<4)$, we find for $60^{\circ}$ TDs in $\mathrm{Si}$ (dashed line in Fig. 2) $\varepsilon_{\mathrm{Si}} d_{\mathrm{Si}}$ $=(8.87 \pm 0.15) \times 10^{-2}$, in surprisingly good agreement with the value of $8.16 \times 10^{-2}$ obtained from our experimental data for the parameter $A$ in Eq. (1). The limiting value of $\varepsilon_{\mathrm{Si}} d_{\mathrm{Si}}$ for the motion of the TDs in the top Si layer as given by Eq. (2) is significantly higher then that for dislocations to cross the $\mathrm{SiGe} / \mathrm{Si}$ interface towards the surface in the intermediate stage of the process.

When the tensile stress force on TD segments in the $\mathrm{Si}$ layer becomes equal to the line tension force, before the relaxation in the SiGe layer has ended, the still advancing TD segments in the SiGe layer produce MD segments at both interfaces bounding the SiGe layer [Fig. 3(b)]. This predicted transition is confirmed by cross-section transmission electron microscopy (XTEM) shown in Fig. 4. The $\mathrm{sSi} / \mathrm{SiGe}$ interface is free of MDs in the case of $6 \mathrm{~nm} \mathrm{Si} / 145 \mathrm{~nm} \mathrm{Si} \sin _{0.72} \mathrm{Ge}_{0.29} / \mathrm{Si}(100)$, but contains a high density of MDs in the case of $15 \mathrm{~nm} \mathrm{Si} / 100 \mathrm{~nm} \mathrm{Si} \mathrm{S}_{0.74} \mathrm{Ge}_{0.26} / \mathrm{Si}(100)$. In addition, stress variations are clearly visible at the $\mathrm{SiGe} / \mathrm{Si}$ substrate interface due to the MDs, but none at the $6 \mathrm{~nm} \mathrm{sSi} / \mathrm{SiGe}$ interface.

For $d_{\mathrm{Si}}>d^{*}$ [Fig. 3(c)], the image force acting on the extending primary loop is no longer able to pull these loops

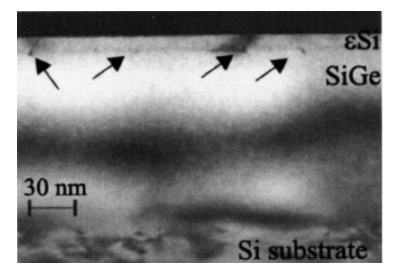

FIG. $6 \mathrm{~nm} \mathrm{sSi} / 145 \mathrm{~nm} \mathrm{Si}_{0.71} \mathrm{Ge}_{0.29} / \mathrm{Si}(100)$ (right) heterostructure. The MDs at the $15 \mathrm{~nm} \mathrm{sSi} / \mathrm{SiGe}$ interface are marked with arrows. This case corresponds to Fig. 3(b).

up to the surface. In this case, at both interfaces of the SiGe layer, two similar MD networks form upon the spreading of TD segments which are now fully confined to the SiGe layer.

According to the above described explanation of our experimental results, strain generation in a thin Si layer by strain relaxation in an epitaxially attached $\mathrm{SiGe}$ buffer layer may be considered as an inverse strain-relaxation process. In contrast to conventional strain relaxation, where dislocations must be nucleated, here dislocations are provided by the $\mathrm{He}$ implantation. This explains why the critical thickness versus the strain relation according to the MB theory is much better fulfilled for strain generation in our Si top layers of medium thickness than for conventional strain relaxation where large discrepancies between theory and experiment are observed.

In conclusion, we have studied strain transfer in a $\mathrm{Si} / \mathrm{SiGe} / \mathrm{Si}(100)$ heterosystem during the relaxation of the $\mathrm{SiGe}$ layer. Dislocations propagating from the SiGe layer into the Si layer establish elastic strain in there. The strain generated depends on the layer thickness and the degree of relaxation of the SiGe layer. We found three thickness regimes. For top $\mathrm{Si}$ thicknesses up to $8 \mathrm{~nm}$ (on $\left.150 \mathrm{~nm} \mathrm{Si} i_{0.74} \mathrm{Ge}_{0.26}\right), 100 \%$ strain transfer is observed. In this case a coherent strained Si layer on the relaxed SiGe layer is formed with only one epitaxial growth step. For intermediate thicknesses the strain buildup is described by inverse strain relaxation, which is well described by the MB theory. The third regime refers to thicknesses beyond a thickness of $\sim 59 \mathrm{~nm}$, where no strain transfer by dislocation propagation through the Si layer is possible.

${ }^{1}$ K. Rim, J. Chu, H. Chen, K. A. Jenkins, T. Kanarsky, K. Lee, A. Mocuta, H. Zhu, R. Roy, J. Newbury, J. Ott, K. Petrarca, P. Mooney, D. Lacey, S. Koester, K. Chan, D. Boyd, M. Ieong, and H.-S. Wong, Symposium on VLSI Technology Digest of Technical Papers 2002, 98.

${ }^{2}$ E. Fitzgerald, M. Currie, S. Samavedam, T. Langdo, G. Taraschi, V. Yang, C. Leitz, and M. Bulsara, Phys. Status Solidi A 171, 227 (1999).

${ }^{3}$ B. Holländer, S. Lenk, S. Mantl, H. Trinkaus, D. Kirch, M. Luysberg, T. Hackbarth, H.-J. Herzog, and P. Fichtner, Nucl. Instrum. Methods Phys. Res. B 175-177, 357 (2001).

${ }^{4}$ H. Trinkaus, B. Holländer, S. Rongen, S. Mantl, H.-J. Herzog, J. Kuchenbecker, and T. Hackbarth, Appl. Phys. Lett. 76, 3552 (2000).

${ }^{5}$ J. Huang, Z. Ye, H. Lu, and D. Que, J. Appl. Phys. 83, 171 (1998).

${ }^{6}$ B. Holländer, R. Butz, and S. Mantl, Phys. Rev. B 46, 6975 (1992).

${ }^{7}$ T. Englert, G. Abstreiter, and J. Pontcharra, Solid-State Electron. 23, 31 (1980).

${ }^{8}$ J. Tsang, P. Mooney, F. Dacol, and J. Chu, J. Appl. Phys. 75, 8098 (1994).

${ }^{9}$ J. Matthews and A. Blakeslee, J. Cryst. Growth 27, 118 (1974).

${ }^{10}$ L. Freund, Adv. Appl. Mech. 30, 1 (1993).

${ }^{11}$ B. Doris, M. Ieong, H. Zhu, Y. Zhang, M. Steen, W. Natzle, S. Callegari, V. Narayanan, J. Cai, S. H. Ku, P. Jamison, Y. Li, Z. Ren, V. Ku, D. Boyd, T. Kanarsky, C. D'Emic, M. Newport, D. Dobuzinsky, S. Deshpande, J. Petrus, R. Jammy, and W. Haensch, Tech. Dig. - Int. Electron Devices Meet. 2003, 631. 